\title{
LIMIAR ANAERÓBIO EM EXERCÍCIOS RESISTIDOS: ANÁLISE DE ASPECTOS METODOLÓGICOS E HEMODINÂMICOS
}

\author{
ANAEROBIC THRESHOLD IN RESISTANCE EXERCISES: ANALYSIS OF METHODOLOGICAL AND \\ HEMODINAMIC ASPECTS
}

Original Articie

ARTIGO ORIGINAL

Artículo Original

\author{
UMBRAL ANAERÓBICO EN EJERCICIOS DE RESISTENCIA: ANÁLISIS DE ASPECTOS \\ METODOLÓGICOS Y HEMODINÁMICOS
}

\author{
Lucas Dantas Maia Forte ${ }^{1}$ \\ (Educador Físico) \\ Fábio Thiago Maciel da Silva' \\ (Educador Físico) \\ Diego Lucena de Medeiros ${ }^{1}$ \\ (Educador Físico) \\ Michelle Sabrina Moreira Silva' \\ (Educadora Física) \\ Gustavo Gomes de Araújo² \\ (Educador Físico) \\ Claudio Luiz de Souza Meireles' \\ (Educador Físico)

\section{Universidade Federal da Paraíba, Laboratório de Estudos do Treinamento Físico Aplicados ao Desempenho e à Saúde, João Pessoa, Paraíba, Brasil. \\ 2. Universidade Federal de Alagoas, Grupo de Pesquisa em Ciência do Esporte, Maceió, Alagoas, Brasil.}

\section{Correspondência:}

Claudio Luiz de Souza Meireles Universidade Federal da Paraíba, Centro de Ciências da Saúde, Departamento de Educação Física, Cidade Universitária, Castelo Branco I, João Pessoa, PB, Brasil. 58000-000. claudiomeireles@hotmail.com

\section{RESUMO}

Introdução: A determinação do limiar anaeróbio em exercícios resistidos tem sido tema de diversos estudos. No entanto, o impacto desta avaliação sobre os parâmetros hemodinâmicos de pressão arterial e frequência cardíaca ainda é desconhecido. Objetivo: Comparar a estimativa do limiar anaeróbio (LAn) obtido em teste e reteste de protocolo incremental nos exercícios resistidos de supino reto (SR) e rosca direta (RD) e analisar o comportamento das variáveis hemodinâmicas de frequência cardíaca (FC), pressão arterial sistólica (PAS) e diastólica (PAD) durante protocolo de cargas incrementais. Métodos: Oito voluntários do sexo masculino praticantes de treinamento resistido foram recrutados e realizaram quatro testes de cargas incrementais (dois no SR e dois na RD) em dias distintos para a determinação do LAn. Durante cada teste, as variáveis de lactato sanguíneo, FC, PAS e PAD foram mensuradas ao final de cada estágio. Resultados: Os valores do LAn expressos em porcentagens da carga máxima (1-RM) para o SR em teste e reteste foram de $19,7 \pm 4,0 \%$ e $18,4 \pm 3,4 \%$ respectivamente e para $\mathrm{RD}$ de $17,7 \pm 3,4 \%$ e $19,4 \pm 3,1 \%$ respectivamente, não sendo identificadas diferenças estatísticas entre estas. No decorrer dos testes, a FC variou em média entre 90 e 135bpm para SR e entre 98 a 150bpm para RD. Apenas a PAS se alterou no decorrer dos testes, variando entre 111,8 a 123,3mmHg no SR e entre 119,4 a 141,3mmHg na RD. Conclusão: Tais resultados nos sugerem que a intensidade relativa ao LAn não é diferente para SR ou RD em teste ou reteste. Não obstante, apesar de diferenças nos comportamentos hemodinâmicos entre os tipos de exercícios (SR vs. RD), os valores de FC e PAS se elevaram dentro de limites clinicamente aceitáveis, como sugerido pela literatura.

Palavras-chave: limiar anaeróbio, treinamento de resistência, ácido láctico, frequência cardíaca, pressão arterial.

\section{ABSTRACT}

Introduction: The anaerobic threshold determination in resistance exercises has been the subject of several studies. However, the impact of these evaluations over the hemodynamic parameters of blood pressure and heart rate are still unknown. Objective: To compare the estimate of the anaerobic threshold (AnT) obtained during test and retest of an incremental protocol in resistance exercises of bench press (BP) and biceps curl (BC), and analyze the hemodynamic variable behavior of heart rate (HR), systolic (SBP) and diastolic blood pressure (DBP) during the incremental load protocol. Methods: Eight male practitioners of resistance training volunteers have been recruited and carried out four incremental load tests (two in BP and two in BC) in distinct days for AnT determination. During each test, the variables of blood lactate, $H R, S B P$ and $D B P$ were measured at the end of each stage. Results: The values of AnT expressed in \% of maximum load (1-RM) for BP in test and retest were 19.7 $\pm 4.0 \%$ and $18.4 \pm 3.4 \%$ respectively, and for $B C$ were $17.7 \pm 3.4 \%$ and $19.4 \pm 3.1 \%$ respectively, not being found statistical differences between these. During tests, HR ranged on average between 90 and $135 \mathrm{bpm}$ for BP and between 98 and $150 \mathrm{bpm}$ for BC. Only the SBP has changed during tests, ranging between 111.8 to $123.3 \mathrm{mmHg}$ in BP and 119.4 to $141.3 \mathrm{mmHg}$ in BC. Conclusion: These results suggest that the intensity related to $A n T$ is not different for $B P$ or $B C$ during test or retest. Despite differences in hemodynamic behavior between exercise types (BP vs. BC), HR and SBP values had an elevation within acceptable clinical limits as suggested by literature.

Keywords: anaerobic threshold, resistance training, lactic acid, heart rate, arterial pressure.

\section{RESUMEN}

Introducción: La determinación del umbral anaeróbico en ejercicios de resistencia, ha sido un tema de diversos estudios, sin embargo, el impacto de esta evaluación sobre los parámetros hemodinámicos de presión arterial y frecuencia cardíaca todavía son desconocidos. Objetivo: Comparar la estimación del umbral anaeróbico (LAn) obtenido en prueba y reprueba del protocolo incremental en los ejercicios de resistencia de press de banca (PB) y curl de bíceps con barra (CB) y analizar el comportamiento de las variables hemodinámicas de frecuencia cardíaca (FC), presión arterial sistólica (PAS) y diastólica (PAD) durante un protocolo de cargas incrementales. Métodos: Ocho voluntarios de sexo masculino, practicantes de entrenamiento de resistencia, fueron reclutados y realizaran cuatro 
pruebas de cargas incrementales (dos en PB y dos en CB) en días distintos para la determinación del LAn. Durante cada prueba, las variables de lactato sanguíneo, FC, PAS y PAD fueron medidas al final de cada fase. Resultados: Los valores de LAn expresados en porcentajes de carga máxima (1-RM) para el PB en prueba y reprueba fueron de $19,7 \pm 4,0 \%$ y $18,4 \pm 3,4 \%$ respectivamentey para CB de $17,7 \pm 3,4 \%$ e 19,4 $23,1 \%$ respectivamente, no siendo identificadas diferencias estadísticas entre estas. En el transcurso de las pruebas, la FC varió, en promedio, entre 90 y $135 \mathrm{bpm}$ para PB y entre 98 a 150bpm para CB. Solamente la PAS se alteró en el transcurso de las pruebas, variando entre 111,8 a $123,3 \mathrm{mmHg}$ en PB y entre 119,4 a 141,3mmHg en CB. Conclusión: Tales resultados nos sugieren que la intensidad relativa al $L A n$ no es diferente para PB o CB en prueba y reprueba. No obstante, a pesar de las diferencias en los comportamientos hemodinámicos entre los tipos de ejercicios (PB vs. CB), los valores de FC y PAS aumentaron dentro de límites clínicamente aceptables como lo sugerido por la bibliografía.

Palabras clave: umbral anaerobico, entrenamiento de resistencia, ácido láctico, frecuencia cardíaca, presión arterial.

\section{INTRODUÇÃO}

Apesar do modelo de exercícios resistidos (ER) ser amplamente utilizado para o desenvolvimento de força, potência e hipertrofia muscular, os benefícios no âmbito da saúde no combate e prevenção de diferentes doenças endócrino-metabólicas foi reconhecido apenas recentemente ${ }^{1-3}$. No entanto, podemos observar na literatura que mesmo nos estudos mais recentes, os métodos utilizados para avaliação e prescrição de treinamento nesta modalidade ainda são comumente baseados em testes de carga máxima ou uma repetição máxima (1-RM) ${ }^{4-6}$, não considerando outros aspectos fisiológicos importantes para a estimação de intensidades específicas de esforço, como por exemplo, variáveis metabólicas e hemodinâmicas.

Neste sentido, alguns trabalhos adaptaram protocolos de cargas incrementais para a determinação do fenômeno do limiar anaeróbio (LAn) em exercícios resistidos ${ }^{3,7-14}$, a fim de estimar a intensidade limítrofe entre a predominância metabólica aeróbia e anaeróbia, a qual é diretamente dependente da capacidade aeróbia do indivíduo em exercício específico ${ }^{15,16}$.

Apesar do LAn em exercícios resistidos representar uma importante abordagem para a avaliação e prescrição de treinamento ${ }^{17,18}$, pouco se sabe a respeito da influência dos aspectos metodológicos sobre a determinação da intensidade do $L A n^{9}$ e principalmente, sobre importantes variáveis hemodinâmicas - como a frequência cardíaca (FC), pressão arterial sistólica (PAS) e diastólica (PAD), no decorrer do teste.

Consequentemente, o presente estudo teve como objetivos: 1) Estimar o LAn por meio da análise da concentração sanguínea de lactato (LAC) durante um protocolo incremental em exercícios resistidos de supino reto (SR) e rosca direta (RD); 2) Verificar se existem diferenças na determinação da intensidade do LAn entre teste e reteste do método proposto; 3 ) caracterizar o comportamento da frequência cardíaca (FC) e pressão arterial sistólica (PAS) e diastólica (PAD) durante o protocolo incremental.

\section{MÉTODOS}

Oito indivíduos do gênero masculino, fisicamente ativos, com experiência mínima de um ano em exercícios resistidos participaram das coletas (tabela 1). Todos os indivíduos foram informados dos riscos e benefícios do estudo e, por conseguinte, responderam negativamente aos itens do questionário PAR-Q (Physical Activity Readiness Questionnaire) ${ }^{19}$. Em seguida, assinaram o Termo de Consentimento Livre e Esclarecido. O estudo foi previamente submetido e aprovado pelo Comitê de Ética em Pesquisa do Centro de Ciências da Saúde - CEP/CCS) da Universidade Federal da Paraíba, PB, Brasil, obedecendo às exigências da Resolução nacional 196/1996, sob número de registro 303/09 e folha de rosto: 297064.

Cada voluntário compareceu ao nosso laboratório durante cinco visitas (com intervalo mínimo de 24h) para a realização dos testes.
Tabela 1. Média \pm DP das características gerais dos participantes do estudo $(n=8)$.

\begin{tabular}{c|c|c|c|c|c|c|c}
\hline & $\begin{array}{c}\text { Idade } \\
\text { (anos) }\end{array}$ & $\begin{array}{c}\text { Massa } \\
(\mathbf{k g})\end{array}$ & $\begin{array}{c}\text { Estatura } \\
\text { (cm) }\end{array}$ & $\begin{array}{c}\text { Gordura } \\
(\mathbf{\%})\end{array}$ & $\begin{array}{c}\text { 1-RM SR } \\
\mathbf{( k g )}\end{array}$ & $\begin{array}{c}\text { 1-RM RD } \\
\text { (kg) }\end{array}$ & $\begin{array}{c}\mathrm{FC}_{\text {max }} \\
\text { (220-idade) }\end{array}$ \\
\hline Média & 21,5 & 81,0 & 176 & 12,4 & 101,5 & 49,7 & 198,5 \\
\hline$\pm \mathrm{DP}$ & 2,3 & 9,8 & 9 & 3,7 & 12,7 & 4,8 & 2,33 \\
\hline
\end{tabular}

Durante a primeira visita, foram realizadas as medidas antropométricas e logo em seguida, a determinação da carga máxima obtida por meio do teste de uma repetição máxima (1-RM) em cada exercício. A densidade corporal dos voluntários foi estimada pela equação de sete dobras proposta por Jackson e Pollock ${ }^{20}$, e o percentual de gordura de acordo com a equação proposta por Siri21. Nos dias subsequentes foram realizados randomicamente quatro testes incrementais para a determinação em teste e reteste do LAn em cada exercício.

\section{Teste de uma repetição máxima (1-RM)}

A determinação da carga máxima para ambos os exercícios (SR e RD) foi realizada no mesmo dia com intervalo mínimo de 20min entre elas. Os procedimentos estiveram de acordo com os reportados anteriormente $e^{9,11}$. Após um aquecimento padrão com carga de aproximadamente 50\% 1-RM, os voluntários realizaram cinco tentativas separadas por um intervalo mínimo de cinco minutos. As cargas das tentativas subsequentes foram acrescidas ou reduzidas de acordo com o sucesso da anterior. A execução realizada com a maior carga pelo indivíduo, sem a possibilidade da realização de uma segunda repetição, foi considerada a carga de 100\% de 1-RM. Com o objetivo de aumentar a precisão do teste, estabeleceu-se uma tolerância máxima de $1 \mathrm{~kg}$ de diferença entre a intensidade de 100\% de 1-RM e a superior a esta.

\section{Protocolo Incremental}

Após uma coleta sanguínea de repouso, iniciou-se o primeiro estágio com a carga de 10\% de 1-RM. Cada estágio teve duração de 3 min com intervalos de 1 min destinados para a coleta sanguínea, medidas de FC, PAS, PAD e incremento de 5\% de 1-RM na carga. A velocidade dos exercícios foi controlada por metrônomo digital o qual estabeleceu uma cadência de 20 repetições por minuto. $O$ final do teste foi determinado mediante a incapacidade do indivíduo de realizar as repetições na cadencia prevista (perder a sincronia com três repetições consecutivas) ou por desistência voluntária.

\section{Determinação do LAn}

O limiar anaeróbio foi determinado pelo método de bissegmentação de regressão linear ${ }^{9}$. Em resumo, duas regressões lineares foram plotadas de forma a se obter a menor soma dos quadrados dos resíduos 
em um gráfico de lactato sanguíneo (eixo y) versus intensidade (eixo x). Ao igualar as equações obtidas para cada regressão, foram derivados os valores de " $x$ " $e$ " $y$ " no ponto de intercessão das retas, representando respectivamente a intensidade do LAn (em \%-1RM) e a concentração sanguínea de lactato referente ao LAn (LAC-LAn). A figura 1 ilustra o método de determinação da intensidade do LAn e o LAC-Lan.

Após assepsia do lóbulo da orelha, as coletas sanguíneas foram realizadas por meio de punção utilizando lanceta de aço inoxidável e descartável. A primeira gota de sangue foi descartada e logo em seguida, $25 \mu$ de sangue foram coletados em capilares de vidro heparinizados e calibrados. Posteriormente o sangue foi transferido para tubos eppendorfs contendo $400 \mu$ de ácido tricloroacético (TCA) à 4\% e armazenados a uma temperatura entre $2^{\circ}$ a $8^{\circ} \mathrm{C}$ para desproteinização do sangue. Posteriormente, foram extraídos $100 \mu \mathrm{L}$ de sobrenadante e transferidos para tubos de ensaio contendo $500 \mu \mathrm{L}$ de reativo (estoque glicina/EDTA e hidrazina-hidrato 1,2ml, 100mg de NAD [Beta-nicotinamide dinicleotide SIGMA] e 150 $\mathrm{L}$ de LDH [L-lactic dehydrogenase bovine heart]) devidamente ajustadas a um $\mathrm{pH}$ de 8,85. As amostras foram lidas a $340 \mathrm{~nm}$ contra a curva de calibração ${ }^{22}$.

Durante o repouso e ao final de cada estágio do teste, as medidas de frequência cardíaca foram realizadas por um frequencímetro (polar SI 800) e a pressão arterial sistólica e diastólica foram aferidas por método auscultatório.

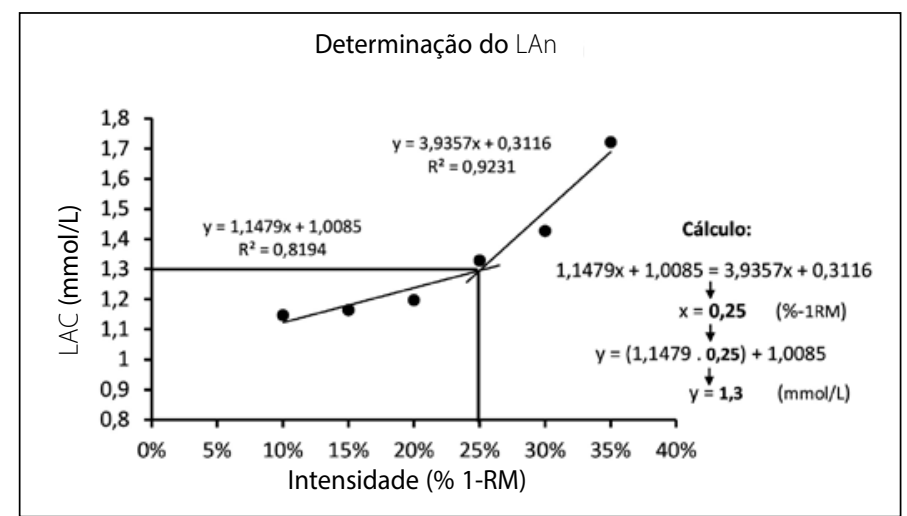

Figura 1. Exemplo da determinação da intensidade (\%-1RM) do limiar anaeróbio (LAn) e da concentração sanguínea de lactato (LAC) durante um protocolo incremental em exercício resistido de rosca direta (RD) pelo método matemático de bissegmentação de regressão linear.

\section{Análise Estatística}

Os dados estão apresentados em média e desvio padrão ( \pm DP). Após confirmação da normalidade dos dados pelo teste de Shapiro Wilk, foi utilizada a análise de variância para amostras repetidas (ANOVA two-way) para a comparação entre as variáveis de intensidade (\%-1RM) e LAC (mmol/L) entre os diferentes momentos (teste e reteste). A análise de variância ANOVA one-way foi utilizada para comparar os valores de FC, PAS e PAD entre os estágios dos protocolos incrementais para os diferentes exercícios (RD e SR). Para medidas de correlação entre as variáveis de intensidade no LAn e tempo total dos testes incrementais, foi utilizado o coeficiente de correlação produto-momento de Pearson. Os dados foram tratados por meio do software Statistica 7.0. Para todas as comparações, adotou-se o nível de significância de 5\%. O poder dos testes estatísticos foi mensurado por meio do software GPower 3.1.7.

\section{RESULTADOS}

Os valores em média \pm DP da intensidade do LAn e das concentrações sanguíneas de lactato em repouso, no LAn e ao final dos testes e retestes incrementais do SR e RD estão dispostos na tabela 2. 0 ANOVA two-way não indicou diferenças significativas para as variáveis abordadas entre teste e reteste e/ou entre os exercícios de SR e RD.

As variáveis hemodinâmicas de FC, PAS e PAD apresentaram comportamento similar entre teste e reteste dos protocolos incrementais no $S R$ e $R D$ (figuras $2 A-B$ e $3 A-B$ respectivamente). A FC se elevou significativamente em relação aos valores de repouso durante os protocolos incrementais em SR ( $p=0,0001)$; tamanho de efeito $=18,75$; poder de teste $=1,0$ ) e RD.

Os coeficientes de produto-momento entre a intensidade do LAn no SR e RD e o tempo total nos respectivos testes não foram estatisticamente correlatos ( $r=0,55$ e 0,50 para SR e RD respectivamente; $p>0,05$ ).

Tabela 2. Média \pm DP da intensidade relativa ao LAn e CSL em repouso, no LAn e ao final do teste.

\begin{tabular}{c|c|c|c|c}
\hline & SR-1 & SR-2 & RD-1 & RD-2 \\
\hline LAn (\%-1RM) & $19,7 \pm 4,0$ & $18,4 \pm 3,4$ & $17,7 \pm 3,4$ & $19,4 \pm 3,1$ \\
\hline LAC-LAn (mmol/L) & $1,8 \pm 0,7$ & $2,0 \pm 0,5$ & $1,7 \pm 0,3$ & $1,6 \pm 0,4$ \\
\hline LAC-Rep (mmol/L) & $1,1 \pm 0,2$ & $1,2 \pm 0,3$ & $1,2 \pm 0,3$ & $1,3 \pm 0,5$ \\
\hline LAC-Final (mmol/L) & $2,8 \pm 0,4$ & $2,7 \pm 0,6$ & $2,9 \pm 0,8$ & $3,2 \pm 1,6$ \\
\hline
\end{tabular}

LAn $(\%-1 R M)=$ intensidade do limiar anaeróbio; $L A C-L A n=$ concentração sanguínea de lactato no LAn; LAC-Rep = concentraçăo sanguínea de lactato em repouso; LAC-Final = concentraçăo sanguínea de lactato ao final do teste; SR-1 = primeiro teste no supino reto; SR-2 = segundo teste no supino reto; RD-1 = primeiro teste na rosca teste; $\mathrm{SR}-1$ = primeiro teste no supino reto; $\mathrm{SR}-2$ -
direta; $\mathrm{RD}-2$ = segundo teste na rosca direta.

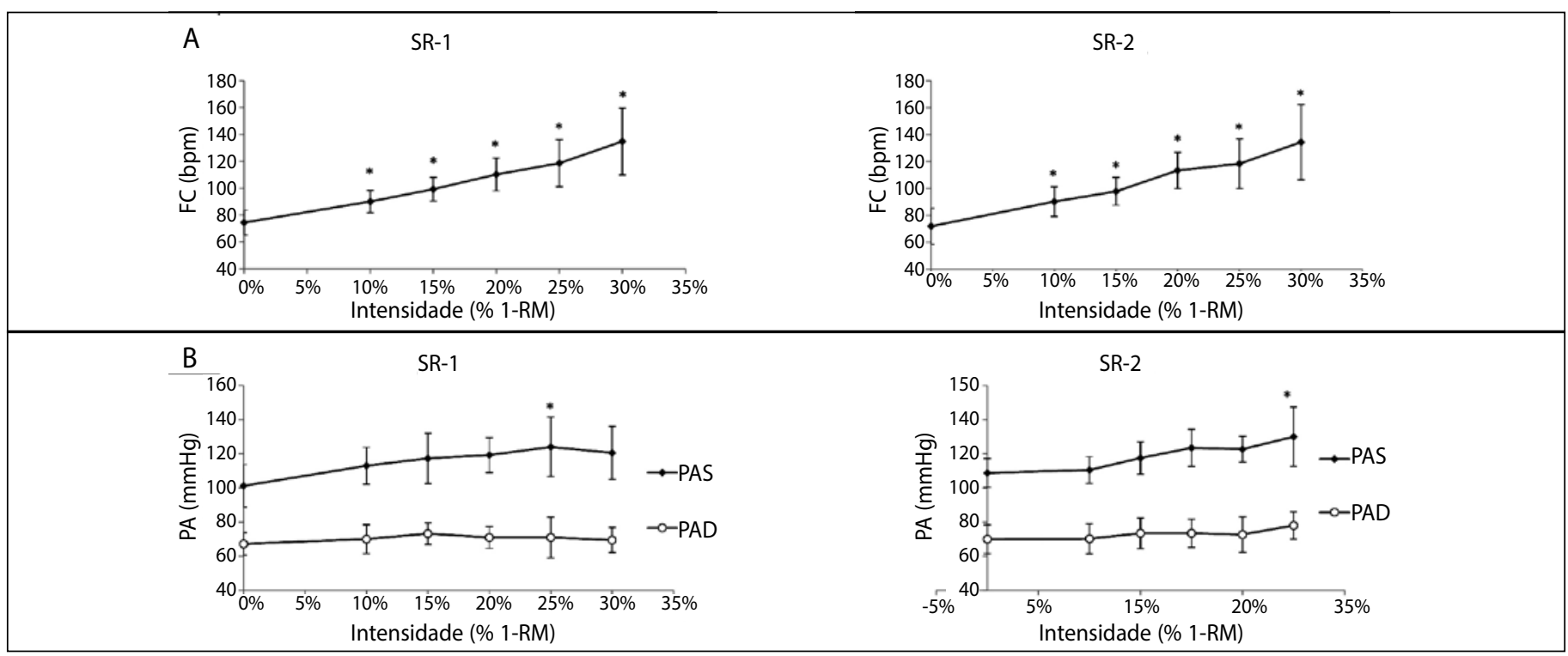

Figura 2. Representação gráfica do comportamento das variáveis hemodinâmicas de frequência cardíaca (A) e pressão arterial (B) durante o protocolo de cargas incrementais no supino reto em teste (SR-1) e reteste $(S R-2) .{ }^{*} p<0,05$ em comparação ao repouso (poder do teste $=1,0$ ). 


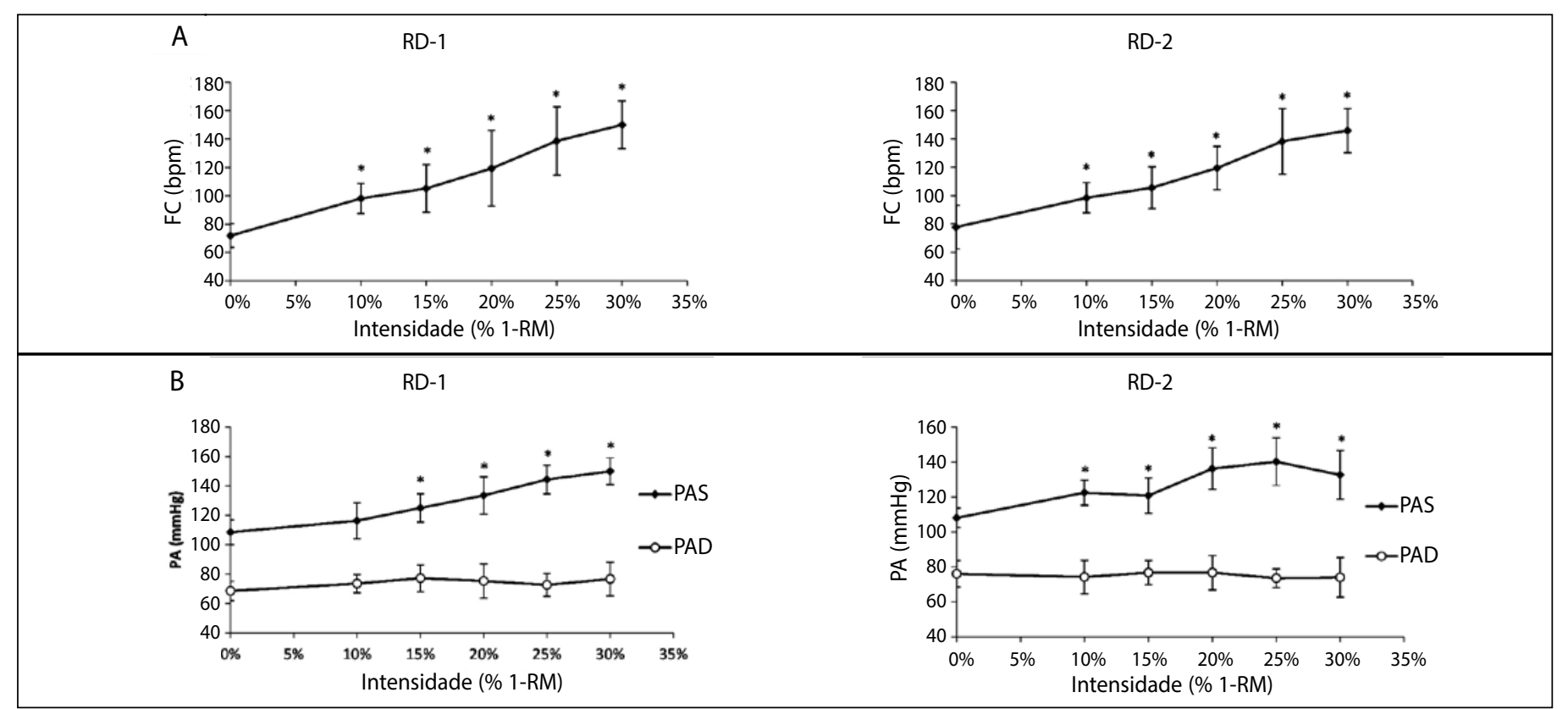

Figura 3. Representação gráfica do comportamento das variáveis hemodinâmicas de frequência cardíaca (A) e pressão arterial (B) durante o protocolo de cargas incrementais na rosca direta em teste (RD-1) e reteste (RD-2).* $p<0,05$ em comparação ao repouso (poder do teste $=1,0$ ).

\section{DISCUSSÃO}

O presente estudo investigou a possibilidade de se determinar o limiar anaeróbio (LAn) nos exercícios resistidos (ER) de supino reto $(\mathrm{SR})$ e rosca direta $(\mathrm{RD})$ durante teste e reteste de um protocolo incremental adaptado ao modelo de ER. De acordo com nossos resultados, o LAn foi identificado em ambos os exercícios em aproximadamente 19\% de 1-RM.

Outros estudos utilizando medidas de lactato e/ou glicose sanguínea durante protocolos incrementais identificaram o LAn em intensidades superiores às do presente estudo (28-36\% 1-RM). Tais diferenças podem estar associadas aos aspectos metodológicos adotados em cada estudo. Os primeiros trabalhos que adaptaram protocolos incrementais para a determinação do LAn em ER utilizaram uma razão de 1 min de esforço para 2 min de pausa entre os estágios do teste incremental ${ }^{11,12}$, enquanto que na presente pesquisa foi utilizada a razão 3 min para cada 1 min de pausa. Apesar de outros trabalhos também terem adotado a mesma razão esforço/pausa abordada nos trabalhos originais ${ }^{7-10,13,14}$, a literatura sugere que quanto menor a razão esforço/pausa durante protocolos incrementais, maior a probabilidade de superestimação do LAn decorrente de uma maior eliminação dos metabólitos produzidos pelo metabolismo anaeróbio, possibilitando o alcance de cargas superiores $^{3,23}$. Esta hipótese se confirma quando observamos que nos trabalhos em que foi utilizada a razão 1/2 (1 minuto de esforço para cada 2 minutos de repouso passivo), os voluntários alcançaram cargas próximas a 70 \%1-RM durante o protocolo incremental, enquanto que na presente pesquisa, a desistência voluntária ocorreu nos estágios entre 25 e 35\% de 1-RM, denotando a ocorrência de anaerobiose sob tais intensidades e impossibilitando a continuação do teste em intensidades superiores. Desta forma, optamos por utilizar razões abordadas classicamente em exercícios cíclicos como em corrida e bicicleta ${ }^{23,24}$. Como resultado, nosso estudo demonstrou uma possível protocolo-dependência da determinação do LAn em ER, principalmente quando manipulada a razão esforço/pausa.

Considerando a falta de estudos sobre a determinação do LAn em $E R$, investigamos a consistência do protocolo proposto por meio de teste e reteste do mesmo. Os valores de LAn apresentados no SR e RD variaram entre 17,7 e 19,7\% 1-RM. Não foram identificadas diferenças significativas entre os tipos de exercício e entre teste e reteste $(p>0,05)$. Resultado similar foi encontrado para as concentrações sanguíneas de lactato de repouso, LAn e ao final de cada teste (tabela 2), sugerindo uma boa consistência do protocolo estudado.

Diversos estudos encontraram relações positivas entre a intensidade relativa ao LAn e o desempenho aeróbio, caracterizando este, como um índice de capacidade aeróbia ${ }^{25,26}$. Assumindo o tempo total do teste como um índice de desempenho, testamos tais correlações com o LAn determinado em SR e RD. No entanto, apesar de se observar uma tendência positiva, o coeficiente de correlação de Pearson não identificou significativas relações entre os parâmetros estudados. Possivelmente este resultado pode ser consequência do reduzido N amostral (oito voluntários), indicando uma limitação da presente pesquisa para esta análise.

Além de caracterizarmos a cinética do lactato durante o protocolo incremental, na presente pesquisa determinamos o comportamento das variáveis hemodinâmicas de frequência cardíaca (FC) e pressão arterial sistólica (PAS) e diastólica (PAD). No decorrer do protocolo incremental a FC aumentou em média de 90 a 135bpm (50 a 67,9\%-FC $C_{\max }$ ) para o

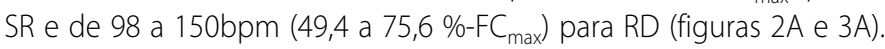
Comportamentos similares foram observados para a PAS em ambos os exercícios, apresentando um aumento proporcional ao incremento das cargas (de 111,8 a $125,3 \mathrm{mmHg}$ no SR e de 119,4 a $141,3 \mathrm{mmHg}$ na $\mathrm{RD}$ ) (figuras $2 \mathrm{~B}$ e $3 \mathrm{~B}$ ). A literatura sugere que tais valores se encontram dentro de um limite de segurança aceitável clinicamente ${ }^{27,28}$. Não obstante, Haslam, McCartney, McKelvie, MacDougall27 sugerem que esforços entre 40\% e 60\% de 1-RM induzem a aumentos da PA dentro de tais limites. Além disso, como demonstrado nas figuras $2 \mathrm{~B}$ e $3 \mathrm{~B}$, não foram encontradas diferenças significativas entre a PAD obtida ao final de qualquer um dos estágios ou em repouso, o que indica a ocorrência de pouca ou nenhuma variação na PAD durante o protocolo incremental para ambos os exercícios. Desta forma, além de poder determinar um importante parâmetro fisiológico em ER, nossos resultados sugerem que o protocolo abordado apresenta uma alta aplicabilidade, possivelmente em diferentes populações.

Curiosamente, os valores de FC e PAS durante o protocolo incremental na RD foram maiores que os obtidos durante o teste no SR. Estas diferenças podem ser explicadas pela realização do SR em decúbito 
dorsal, a qual favorece o retorno venoso por reduzir o tônus simpático via barorreceptores do seio carotídeo e consequentemente, reduzindo a FC e PAS durante o exercício ${ }^{29}$.

Curiosamente, foram observadas baixas concentrações de lactato sanguíneo durante os testes incrementais em ambos os exercícios (tabela 2), principalmente para a LAC-LAn (entre 1,6 e 2,0 mmol/L). Muitos estudos reportam concentrações sanguíneas de lactato relativas ao $L$ An por volta de $4 \mathrm{mmol} / \mathrm{L}^{23}$ independente do nível de treinamento ${ }^{26}$. Estas diferenças provavelmente estão relacionadas ao grupamento muscular utilizado nos exercícios, bem como à quantidade total de massa muscular recrutada. Ao contrário dos trabalhos que comumente utilizam exercícios de membros inferiores com grandes grupos musculares como corrida e ciclismo, o presente estudo determinou o LAn em exercícios de membros superiores com menor massa muscular e consequentemente, menor capacidade de produção de lactato. Ainda, estudos prévios determinaram LAn em exercícios resistidos e também encontraram valores de concentração sanguínea de lactato no LAn semelhantes aos da presente pesquisa9, 11, suportando a hipótese de que o LAC-LAn é dependente do grupo muscular exercitado.

\section{CONCLUSÃO}

Concluímos que o LAn em exercícios resistidos de RD e SR pode ser determinado via LAC durante o protocolo incremental proposto na presente pesquisa. Além disso, os valores de LAn obtidos entre teste e reteste do referido protocolo não diferiram entre si. A cinética das variáveis hemodinâmicas oscilou dentro de limites aceitáveis para a aplicação na população estudada, e possivelmente, indicando um protocolo de boa aplicabilidade para a população idosa e/ou portadores de doenças endócrino-metabólicas.

Todos os autores declararam não haver qualquer potencial conflito de interesses referente a este artigo.

\section{REFERÊNCIAS}

1. Pollock ML, Evans WJ. Resistance training for health and disease: introduction. Medicine and science in sports and exercise. 1999; 31(1):10-1.

2. Pollock ML, Franklin BA, Balady GJ, Chaitman BL, Fleg JL, Fletcher B, et al. AHA Science Advisory. Resistance exercise in individuals with and without cardiovascular disease: benefits, rationale, safety, and prescription: An advisory from the Committee on Exercise, Rehabilitation, and Prevention, Council on Clinical Cardiology, American Heart Association; Position paper endorsed by the American College of Sports Medicine. Circulation. 2000;101(7):828-33.

3. Fontes-Carvalho R, Azevedo Al, Sampaio F, Teixeira M, Bettencourt N, Campos L, et al. The Effect of Exercise Training on Diastolic and Systolic Function After Acute Myocardial Infarction: A Medicine (Baltimore). 2015;94(36):e1450.

4. Chen YM, Zhu M, Zhang YX. Combined endurance-resistance training improves submaximal exercise capacity in elderly heart failure patients: a systematic review of randomized controlled trials. Int J Cardiol. 2013;166(1):250-2.

5. Laoutaris ID, Adamopoulos S, Manginas A, Panagiotakos DB, Kallistratos MS, Doulaptsis C, et al. Benefits of combined aerobic/resistance/inspiratory training in patients with chronic heart failure. A complete exercise model? A prospective randomised study. Int J Cardiol. 2013;167(5):1967-72.

6. Yardley JE, Kenny GP, Perkins BA, Riddell MC, Balaa N, Malcolm J, et al. Resistance versus aerobic exercise: acute effects on glycemia in type 1 diabetes. Diabetes Care. 2013;36(3):537-42.

7. Simoes RP, Castello-Simoes V, Mendes RG, Archiza B, Dos Santos DA, Bonjorno Jr JC, et al. Identification of anaerobic threshold by analysis of heart rate variability during discontinuous dynamic and resistance exercise protocols in healthy older men. Clin Physiol Funct Imaging. 2014;34(2):98-108.

8. de Sousa NM, Magosso RF, Pereira GB, Souza MV, Vieira A, Marine DA, et al. Acute cardiorespiratory and metabolic responses during resistance exercise in the lactate threshold intensity. Int J Sports Med. 2012;33(2):108-13.

9. de Sousa NM, Magosso RF, Pereira GB, Leite RD, Arakelian VM, Montagnolli AN, et al. The measurement of lactate threshold in resistance exercise: a comparison of methods. Clin Physiol Funct Imaging. 2011;31(5):376-81.

10. Rocha RM, Bomfim DL, Nascimento TBR, Moreira SR, Simoes HG. A variação do método de incremento de cargas não altera a determinação do limiar de lactato em exercício resistido. Rev Bras Med Esporte. 2010;16(4):282-5

11. de Oliveira JC, Baldissera V, Simoes HG, Aguiar AP, Azevedo PHSM, Poian PAF, et al. Identification of the lactate threshold and the blood glucose threshold in resistance exercise. Rev Bras Med Esporte. 2006;12(6):333-8

12. de Barros CLM, Agostini GG, Garcia ES, Baldissera V. Limiar de lactato em exercício resistido. Motriz. 2004;10(1):31-6.

13. Garnacho-Castano MV, Dominguez R, Ruiz-Solano P, Mate-Munoz JL. Acute Physiological and Mechanical Responses During Resistance Exercise at the Lactate Threshold Intensity. J Strength Cond Res. 2015 Oct;29(10):2867-73
14. Garnacho-Castano MV, Dominguez R, Mate-Munoz JL. Understanding the Meaning of Lactate Threshold in Resistance Exercises. Int J Sports Med. 2015 Mar 26. [Epub ahead of print].

15. Faude O, Kindermann W, Meyer T. Lactate threshold concepts: how valid are they? Sports Med. 2009; 39(6):469-90.

16. Svedahl K, Maclntosh BR. Anaerobic threshold: the concept and methods of measurement. Can Appl Physiol. 2003;28(2):299-323

17. Cunha VCN, Cunha RR, Segundo PR, Moreira SR, Simoes HG. Treinamento de natação na intensidade do limiar anaeróbio melhora a aptidão funcional de ratos idosos. Rev Bras Med Esporte. 2008;14:538-43

18. Gordon NF, Scott CB. Exercise intensity prescription in cardiovascular disease. Theoretical basis for anaerobic threshold determination. J Cardiopulm Rehabil. 1995;15(3):193-6.

19. Cardinal BJ, Esters J, Cardinal MK. Evaluation of the revised physical activity readiness questionnaire in older adults. Med Sci Sports Exerc. 1996;28(4):468-72.

20. Jackson AS, Pollock ML. Generalized equations for predicting body density of men. Br J Nutr. 1978;40(3):497-504.

21. Siri WE. Body composition from fluid spaces and density: analysis of methods. 1991. Nutrition. 1993;9(5):480-91.

22. Engel PC, Jones JB. Causes and elimination of erratic blanks in enzymatic metabolite assays involving the use of NAD+ in alkaline hydrazine buffers: improved conditions for the assay of L-glutamate, L-lactate, and other metabolites. Anal Biochem. 1978;88(2):475-84.

23. Heck H, Mader A, Hess G, Mucke S, Muller R, Hollmann W. Justification of the 4-mmol/l lactate threshold. Int J Sports Med. 1985;6(3):117-30.

24. Yoshida T. Effect of exercise duration during incremental exercise on the determination of anaerobic threshold and the onset of blood lactate accumulation. Eur J Appl Physiol Occup Physiol. 1984;53(3):196-9.

25. Meyer T, Lucia A, Earnest CP, Kindermann W. A conceptual framework for performance diagnosis and training prescription from submaximal gas exchange parameters--theory and application. Int J Sports Med. 2005;26(Suppl 1):S38-48.

26. Beneke R, Hutler M, Leithauser RM. Maximal lactate-steady-state independent of performance. Med Sci Sports Exerc. 2000;32(6):1135-9.

27. Haslam DR, McCartney SN, McKelvie RS, MacDougall JD. Direct measurements of arterial blood pressure during formal weightlifting in cardiac patients. J Cardiopul Rehabil. 1988;8:213-25.

28. Harris KA, Holly RG. Physiological response to circuit weight training in borderline hypertensive subjects. Med Sci Sports Exerc. 1987;19(3):246-52.

29. Kruszewski P, Bieniszewski L, Neubauer-Geryk J, Swierblewska E, Krupa-Wojciechowska B. Supine body position is an important factor influencing postprandial ambulatory blood pressure. Med Sci Monit. 2003;9(1):CR34-41. 